\title{
MANIFESTAÇÕES NEUROLÓGICAS EM CRIANÇAS E ADOLESCENTES INFECTADOS E EXPOSTOS AO HIV-1
}

\author{
Cristiane Rocha', Aída Gouvêa², Daisy Machado³, Kelly Cunegundes4, \\ Suênia Beltrão ${ }^{4}$, Fabiana Bononit, Regina Célia Succi ${ }^{5}$
}

\begin{abstract}
RESUMO - O envolvimento do sistema nervoso central SNC na infecção pelo HIV-1 em crianças pode estar evidente desde o início ou demorar muitos anos para se manifestar. Microcefalia, rebaixamento cognitivo, sinais piramidais, distúrbios do humor e do comportamento e complicações pelo uso da terapia antiretroviral são comuns. Este é um trabalho observacional, descritivo e seccional aja finalidade é descrever as alterações do exame neurológico em um grupo de crianças e adolescentes expostos pelo HIV-1 durante o período perinatal. Foram avaliados 173 pacientes. Muitos pacientes tinham superposição de alterações de exame neu rológico e/ou mais de um diagnóstico. As alterações mais comunsforam: re t a rdo do desenvolvimento neuropsicomotor, atraso de linguagem, deficiência mental, síndrome piramidal, hiporreflexia. 0 exame neurdógico foi alterado em $67 \%$ dos casos, mesmo naqueles pacientes soro-revertidos. Sugerimos que existe alto risco para doença neurológica nesse grupo de pacientes e que a prog ressão da infecção pelo HIV-1 acentua o aparecimento de co-morbidades e comprometimento de seu prognóstico.
\end{abstract}

PALAVRAS-CHAVE: HIV-1, sistema nervoso, exame neurológico.

\begin{abstract}
Neurological findings in a group of children and adolescents exposed and infected by HIV-1
ABSTRACT - The CNS infection by HIV-1 in infancy could be present immediately after infection or became manifest later. Microcephalia, mental ret a rdation, pyramidal signs, humor and behavioral disorders and a nt i ret roviral therapy complications are common. This is an observational, sectional and descriptive study about findings on neurological examination of 173 patients in a group of children and adolescentsinfected and exposed to HIV-1 in perinatal period. Most of them had more than one neurological finding or different diagnosis. The more common findings were: encephalopathy, mental retardation, language delay, pyramidal signs, hyporreflexia. The neurological examination was abnormal in $67 \%$ of all patients even in sororeverters. We sugest that this group has a high risk to neurological disease and the development of co-morbidity is directly correlated to dinical deterioration by HIV-1 infection.
\end{abstract}

KEY WORDS: HIV-1,nervous system, neurological examination.

Durante os primeiros anos da epidemia de SIDA, a encefalopatia foi considerada uma das manifestações preco ces da doença, acreditando-se que era mais comum na infância. Atualmente, apóso estudo de duas grandes coortes, uma francesa ${ }^{1}$ e outra americana², verificou-se que a pro g ressão dos sintomas definidores de SIDA no primeiro ano de vida era de $19 \%$ e $4,5 \%$ ao ano, respectivamente e, a posteriori, muito menor do que descrita no passado, p rovavelmente devido ao diagnóstico precoce da infecção e conseqüente intervenção terapêutica ${ }^{3,4}$.

No lactente jovem, a encefalopatia pelo HIV-1 é, provavelmente, conseqüência direta da ação do vírus em um cérebro em desenvolvimento, o que poderia justificar a diferença de evolução dínica entre adultos e crianças. As manifestações sistêmicas da doença nem sempre cursam com comprometimento simultâneo do sistema nervoso central (SNC) e a expressão clínico-neurológica é bastante variável ${ }^{3}$.

As conseqüências do envolvimento do SNC na infecção pelo HIV-1 em crianças podem estar evidentes desde o início do quadro dínico ou demorar muitos anos para se manifestar e, nesse caso, sobrevêm associadas à piora do estado dínico-imunológico do paciente, o que aumenta a importância de um diagnóstico precoce 5 . Além de complicações pelo uso da terapia antiretroviral, as manifestações neurológicas da infecção pelo HIV-1 mais freqüen-

\footnotetext{
${ }^{1} \mathrm{~N}$ e u ropediatra do Hospital Santa Marcelina e Ambulatório de AIDS-Infectopediatria, Universidade Federal de São Paulo, SP, Brasil (UNIFESP); ${ }^{2}$ Mestre em Pediatria e Médica do Ambulatório de AIDS-Infectopediatria-UNIFESP; ${ }^{3}$ Doutor em Medicina e Médica Assistente do Ambulatório de AIDS-Infectopediatria-UNIFESP; ${ }^{4}$ M édica do Ambulatório de AIDS-Infectopediatria-UNIFESP; ${ }^{5}$ Professora Adjunta da Disciplina de Infectologia - Departamento de Pediatria - UNIFESP.
}

Recebido 22 Fevereiro 2005, recebido na forma final 10 Maio 2005. Aceito 15 Junho 2005.

Dra. Cristiane Rocha - Rua Nicolau S. Queiroz 406/42 - 04105-001 São Paulo SP - Brasil. E-mail: cri.rocha2@ig.com.br 
tes na infância são: microcefalia, rebaixamento cognitivo, encefalopatia, sinais piramidais, distúrbios do humor e do comportamento ${ }^{4-6}$.

Este é um estudo observacional, ret rospectivo e seccional, aprovado pelo Comitê de Ética, cujo o bjetivo é descrever as alterações no exame físico neurológico tradicional em um grupo de crianças e adolescentes infectados e/ou expostos ao HIV-1 no período perinatal.

\section{MÉTODO}

Entrejaneiro de 2000 e junho de 2003, 652 crianças expostas e/ou infectadas pelo HIV-1 estavam em acompanhamento no Ambulatório de AIDS pediátrica da UNIFESP - CEADIPe. Desse grupo, 173 crianças foram avaliadas por um único neuropediatra, através do exame neurdógico tradicional como parte do acompanhamento multidisciplinar rotineiro do serviço, ou seja, quando o pediatra acompanhante suspeitava de alguma alteração neurológica o paciente era encaminhado para avaliação com o neuropediatra. O acompanhamento dínico multidisciplinar indicava reavaliação posterior ou não. Como recurso complementar, foram utilizados também os critérios do DSM-IV-R e quando necessário, os pacientes eram encaminhadas para avaliação com outras especialidades, tais como fonoaudiologia ou psicologia. Cada criança fez pelo menos duas consultas com neuropediatra, exceto as não infectadas que foram incluídas mesmo aquelas com apenas uma avaliação (ex: hipotonia nos dois primeiros anos de vida superada).

As 173 crianças avaliadas foram divididas em 3 grupos:

Grupo 1 - constituído por 25 criançasnasadas de mães infectadas pelo HIV-1 e, portanto, expostas ao vírus no período perinatal. No seguimento dínico posterior foi possível afastar a infecção, nesse caso chamadas de sorrevertidas.

Grupo 2 - constituído por 54 crianças definitivamente infectadas pelo HIV e que não apresentavam sinais ou sintomas da infecção (dlassificação dínica "N") ou apresentavam sinais e sintomas leves de infecção (classificação " $A$ ");

G rupo 3 - constituído por 94 crianças infectadas pelo HIV com sinais e sintomas moderados (dassificação dínica "B") ou graves (classificação dínica "C") da doença.

\section{RESULTADOS}

As 173 pacientes foram classificadasquanto ao sexo e idade na primeira avaliação (Tabela 1). A distribuição por sexo foi similar nos grupos 2 e 3 , tendo havido diferença apenas no grupo 1 onde os meninos corresponderam a $68 \%$ da amostra.

O resultado do exame neurológico pode ser visto na Tabela 2. 54 pacientes $(30,6 \%)$ avaliados tinham mais de um diagnóstico ou achado clínico neurológico (ex: retardo do desenvolvimento neuro-
Tabela 1. Distribuição dos pacientes quanto à idade e sexo.

\begin{tabular}{lccc}
\hline & Grupo $1^{\mathrm{a}}$ & Grupo $2^{\mathrm{b}}$ & Grupo $3^{\mathrm{c}}$ \\
\hline Total & 25 & 54 & 94 \\
Sexo Masculino & 17 & 22 & 48 \\
Sexo Feminino & 8 & 32 & 47 \\
Idade (meses) & $1-24$ & $12-180$ & $12-180$ \\
\hline
\end{tabular}

a, Grupo 1: crianças nascidas de mães HIV-1+, não infectadas (SR); $b$, Grupo 2: assintomáticos (classe N) e paucissintomáticas(A); c, Grupo 3: sintomas moderados (classe B) ou sintomas graves (classe C).

Tabela 2. Distribuição segundo os grupos e achados no exame neurológico.

\begin{tabular}{lccc}
\hline & $\begin{array}{c}\text { Grupo 1 } \\
(n=25)\end{array}$ & $\begin{array}{c}\text { Grupo 2 } \\
(n=54)\end{array}$ & $\begin{array}{c}\text { Grupo 3 } \\
(n=94)\end{array}$ \\
\hline Normal $(n-\%)$ & $11-44 \%$ & $25-46,3 \%$ & $18-19 \%$ \\
Anormal $(n-\%)$ & $14-56 \%$ & $29-53,7 \%$ & $76-81 \%$ \\
\hline
\end{tabular}

psicomotor (RDNPM) prévio e deficiência mental (DM) anos depois) ou superposição de alterações de exame neurológico (ex: hiperreflexia em membros superiores, reflexos profundos abolidos em um membro inferior e normal no contralateral), dificultando a definição exata do diagnóstico principal. A única exceção era para aqueles pacientes que foram diagnosticados como normais por não nenhuma alteração preg ressa ou atual seja na sua anamnese ou em seu exame físico neurológico.

As crianças com alguma alteração no exame n eu rológico $(115 / 173=66,5 \%)$ estavam assim distribuídas: 14 crianças do grupo 1 (14/25 - 56\%), 29 crianças do grupo 2 (29/54 - 53,7 \%) e em 76 crianças do grupo 3 (76/95 - 80\%).

Os achados anormais mais comuns entre todas as crianças avaliadas foram: hiporreflexia (41casos/115 - 35,6\%), retardo do desenvolvimento neuropsicomotor atual ou pregresso do RDNPM (22 casos/115 - 19\%), atraso de linguagem - AL (21casos/115 - 19\%), deficiência mental - DM (11 casos/115 - 9,5\%), síndrome piramidal (11 casos/115 - 9,5\%), paralisia cerebral - PC (4 casos/115 - 3,5\%).

Todas as crianças do grupo 1 foram expostas ao HIV durante a gestação e no seguimento clínico foi possível afastar a infecção pelo vírus. Nesse grupo, 3 crianças apresentaram hipotonia na primeira avaliação dentro do primeiro ano de vida, com normalização desse quadro na segunda avaliação, 3 a 6 meses depois. Três pacientes foram prematuros e desses dois evoluíram clinicamente como PC, e o tercei ro com hipotonia no primeiro ano de vida e com normalização posteriori. Havia três pacientes com RDNPM, um tinha um quadro de displasia es- 
quelética e nos outros não foi achado causa que explicasse $o$ atraso. Encontramos três pacientes, dois meninose uma menina com convulsão febril e desenvolvimento normal (2/115 - 1,7\%). Havia ainda: um paciente com macrocrania e outro com quadro piramidal leve, ambos com DNPM normais. Os demais, 10 pacientes, eram normais e tiveram alta entre 2 e 3 anos de idade com todos os exames nomais e desenvolvimento neurológico adequado.

No grupo 2 encontramos 24 (24/54 - 44\%) pacientes com anamnese e exame neurológico normal durante todo o acompanhamento clínico. No entanto, houve um número maior de afecções neu rológicas associadas, sendo as mais freqüentes: hiporreflexia (15/54 - 27,7\%), atraso de linguagem (11/54 -20,4\%), deficiência mental (5/54 - 9,2\%) e retardo do desenvolvimento $(4 / 54-7,4 \%)$. Apenas dois pacientes cursaram com epilepsia parcial (uma menina de 13 anos com crises parciais recentes e 1 menino com crises parciais desde 6 anos de idade).

O grupo 3 foi o mais numeroso, contando com 94 de crianças (94/173 - 54,3\%) das quais apenas $18 \%$ (17/94 - 18\%) apresentavam exame neuro lógico normal e $82 \%$ (17/94 - 82\%) apresentavam uma ou mais anormalidades clínicas. Os achados mais comuns foram a hiporreflexia (23/94 -24,5\%), RDNPM (16/94 - 17\%) e AL (10/94 - 10,6\%). Tivemos ainda $4(4 / 115$ - 3,4\%) crianças que iniciaram o quad ro de imunodeficiência com AVC de cerebral média ${ }^{7}$. Além disso, encontramostambém como doença associada e não secundária ao HIV-1, 3 crianças com hiperatividade, uma menina com quadro de psicose infantil e um adolescente com depressão, todos sendo apenas tratados com psicoterapia.

Necessário alertar que pacientes com hiporreflexia estão em fase de esclarecimento diagnóstico, se o quadro é de ordem neuropática ou miopática, por isso foi decidido informar apenas como achado de alteração de exame físico, sem correlacionar com o uso de terapia antiretroviral ou como ação direta do HIV sobre o sistema neuromuscular.

\section{DISCUSSÃO}

Segundo Maccmillan e colaboradores ${ }^{7}$, o cérebro é o primeiro alvo na infecção pelo HIV-1, o que comumente leva a uma redução do crescimento cerebral ou um quadro de regressão global ${ }^{8}$. Crianças com infecção adquirida via materna (infecção vertical) costumam ser mais af etadas do ponto de vista motor e cognitivo, comparadas com aquelas só expostas ou infectadas no período intraparto, provavelmente pela longa exposição aos efeitos do vírus intra-útero ${ }^{3,5,9,10}$. Sabemos, ainda, que esses pacientes em geral provêem de uma gestação de risco pela própria condição materna da infecção pelo HIV-1, independente dessas mães terem feito uso de terapia antire troviral corretamente. Osfato res ambientais negativos e outras co-morbidades desfavoráveis são igualmente importantes nesse contexto. Além disso, a falta de estímulo social e alimentação saudável para a criança no primeiro ano de vida contribui ainda mais na morbidade geral ${ }^{6,9}$.

Nesses dois grupos as alterações neurológicas encontradas eram semelhantes, com uma pequena diferença: ocorreu discreta predominância de alterações cognitivas no grupo 2 (AL- 11 casos + DM 5 casos) em relação ao grupo 3, em que predominou um quadro motor periférico (hiporreflexia 23 casos) e central (síndrome piramidal - 8 casos, o RDNPM - 16 casos, que pode ter sido preg resso ou recente; PC - 2 casos, seqüela de AVC - 4 casos).

Lea n d ro-Mehri e colaboradores estudaram em Campinas a curva de peso e crescimento de 71 crianças infectadas por transmissão vertical e verificaram a ocorrência de falência do crescimento em $30 \%$ nesse grupo, gerando uma menor sobrevida. Atribuem, ainda, às condições sócio-econômicas, um fator importante para co-morbidades ${ }^{11}$.

Tellechea-Rotta e Legido, em nosso meio, acompanharam 340 crianças infectadas pelo HIV-1 por transmissão vertical e encontraram que 57\% apresentavam algum tipo de sintoma neurológico ${ }^{12}$.

Um estudo prospectivo feito em Curitiba, Bruck e colaboradore $s^{13}$ acompanharam durante 5 anos 83 crianças expostas ao HIV-1. Observaram alterações neurológicas moderadas em $55 \%$ das crianças infectadas. No grupo de crianças expostas ao vínus e não infectadas (ou soro-revertidas) esse comprometimento chegou a 40\%.Concluem que o HIV-1 é um agente causador de encefalopatia precoce na infância.

Tardieu e colaboradores estudaram crianças com encefalopatias diagnosticadas durante o estudo francês SOROCO ${ }^{1,3}$. Afirmaram que os quadro s de encefalopatia precoce, que ocorrem dentro do primei roano de vida, são decorrentes de eventos do período fetal tardio, provavelmente, pela pouca proteção dada pelo AZT prescrito tardiamente na gravidez. Talvez isso explique, de alguma forma, as alterações encontradas no grupo 1 (56\% de anormalidades) e que com a soro-conversão e normalização do quadro clínico e imunológico, essas crianças tenham superado os problemas iniciais. 
A hiperreflexia foi umachado comum no grupo 2 , talvez explicado pela presença do vírus em diferentesáreas do cérebro, predominando nas regiões dos núcleos caudado, substância branca profunda e temporal, como demonstrou McClernon em 2001'14.

Por outro lado, a hiporreflexia, foi o achado mais comum no grupo 3, em crianças com quadro clínico mais grave e mais crônico. A lenta evolução da doença e o uso da terapia antiretroviral potente podem causar, alem das complicações sistêmicas conhecidas, eventos deletérios paralelos a longo prazo, como as neuropatias e as miopatias ${ }^{5,6,11,14}$. Segundo Schiffito e colaboradores ${ }^{15}$ a polineuropatia sensitiva distal é a causa mais comum em pacientes infectados pelo HIV-1, afetando em torno de $35 \%$ dos pacientes adultos, sendo decorrente tanto da ação do vírus como da ação tóxica de drogas como a didanosídeo e a estavudina. No Rio de J aneiro, Pruffer estudou a velocidade de condução nervosa em 39 crianças infectadas pelo HIV-1, mai o resde 5 anos. Constatou sinais e sintomas de neuropatia periférica do tipo axonal em $34 \%$ dos casos, semelhante aos achados em adultos ${ }^{16}$.

A variedade de alterações neurológicas encontradas em nosso grupo de pacientes, seja na história clínica, no exame físico e no diagnóstico final da criança infectada e/ou exposta pelo HIV-1 é muito grande e muito difícil de sistematizar. Se o uso do AZT, na gestante infectada e durante o período intraparto, reduziu o risco de transmissão vertical do ví nus e da presença de encefalopatia no primeiro ano de vida da criança, a indusão dosinibidores de p rotease modificou o perfil da evolução da SIDA nesse grupo, melhorando sua sobrevida. Além disso, observou-se o surgimento de mudanças nos tipos de doenças pediátricas mais comuns, dando espaço para entidades nosológicas de caráter mais crônico.

Em conclusão, o grupo por nós estudado é um grupo de alto risco para doença neurológica, por ter $67 \%$ da amostra comprometida. À medida que a dassificação clínica dos pacientes progride em gravidade, ocorre aumento de co-morbidade para doenças neurológicas. Acreditamos que o acompanhamento neurológico freqüente pode identificar alterações precocemente, com a intenção de intervenção e reabilitação paralelas ao tratamento medicamentoso, cujo objetivo final é melhorar a qualidade de vida desses pacientes.

\section{REFERÊNCIAS}

1. Blanche S, Newell ML, Mayaux MJ, et al. Morbidity and mortality in European children vertically infected by HIV-1. J Acquir Immune Defic Syndr 1997;14:442-450.

2. Pliner V, Weedon J, Thomas PA, et al, and the New York City Perinatal HIV Transmission Collaborative Study Group. Incubation period of HIV-1 in perinatally infected children. AIDS 1998;12:759-766.

3. Tardieu M, Le Chenadec J, Persoz A, et al. HIV-1 related encephalopathy in infants compared with children and adults. Neurology 2000;54:1089-1095.

4. Gisslén M, Hagberg L. Antiretroviral treatment of central nervous sistem HIV-1 infection : a review. HIV Medicine 2001;2:97-104.

5. Belman AL. Pediatric Neuro-AIDS: update. Syllabus of American Academy of Neurology, 2002.

6. Brew JB. HIV Neurology update. Syllabus of American Academy of Neurology, 2003.

7. Macmillan C, Magder LS, Brouwers $\mathrm{P}$, et al. Head growth and neurodevelopment of infants born to HIV-1-infected drug-using women. Neurology 2001;57:1402-1411.

8. Smith R, Malee K, Charurat M. Timing of perinatal human immunodeficiencyvirus type 1 infection and rate of neurodevelopment. The woman and infant transmission study group. Pediatr Infect Dis J 2000; 19:862-871.

9. Gay CL, Armstrong DF, Cohen D, et al. The effects of HIV on cognitive and motor development in children born to HIV-seropositive women with no reported drug use: birth to 24 months. Pediatrics, 1995;93:1078-1082.

10. Rocha C, Gouvêa ATB, Machado D, et al.Infarto cerebral em duas crianças infectadas pelo HIV-1. Arq Neuropsiquiatr 2003;61:1015-1017.

11. Le and ro-Mehri VA, Vilela MMS, Silva MN, Barros AA Filho. Características do crescimento de crianças infectadas com o vírus da imunodeficiência humana. Pediatria (São Paulo) 2001;1:17-26.

12. Tellechea-Rotta N, Legido A. Acquired immunodeficiency syndrome by vertical transmission: neurological disorders. Rev Neurol 2003;36:255-263.

13. Bruck I, Tahan TT, Cruz CR, et al. Developmental milestones of vertically HIV infected and seroreverters children. Follow-up of 83 children. Arq Neuropsiquiatr 2001;59:691-695.

14. McClernon DR, Lanier R, Gartner R, et al. HIV in brain. RNA levels and patterns of zidovudine resistence. Neurology 2001;57:1396-1401.

15. Schiffitto G, McDermont MP, McArthur JC, et al. Incidence of and risk factors for HIV-associated distal sensory polineuropathy. Neurology 2002;58:1764-1768.

16. Araujo APQC. Peripheral neuropathy in immunodeficiency virus (HIV) infected children. Arq Neuropsiquiatr 1999;57:709. 